\title{
Programming and execution of sequential movements in Parkinson's disease
}

\author{
ROBERT D RAFAL, * ALBRECHT W INHOFF, $\dagger$ JOSEPH H FRIEDMAN,* \\ EMILY BERNSTEIN*
}

From the Division of Neurology,* Roger Williams General Hospital, and Brown University, Providence, Rhode Island, and the Department of Psychology, + University of New Hampshire, Durham, New Hampshire, USA

SUMmaRY In separate blocks of a simple reaction time (RT) task, eight Parkinsonian and eight control subjects executed finger press sequences with one (index finger), two (index finger-ring) or three (index finger-ring-middle) components. Programming was inferred from the increase latency to intiate the first component as a function of the length of the entire sequence; and from the systematic decrease in inter-response latencies for the second and third components. Overall RT was slower in the Parkinsonians but the programming effects were comparable in the two groups. Intact basal ganglia function appears not to be necessary for programming sequential finger movements, or retrieving subprograms for execution.

The dramatic impairment in motility suffered by patients with Parkinson's disease calls special attention to the role of the basal ganglia in the control of movement. Among the symptoms of Parkinsonism, however, the slowness in initiating movement, as measured by an increase in reaction time (RT) to begin, remains poorly understood. Angel et al demonstrated that the increased latency for initiating responses (RT) could not be attributed solely to weakness, rigidity or more peripheral, mechanical limitations, and argued for a disorder at "the highest levels" 1 of motor control. The current investigation explores the hypothesis that the basal ganglia functions in the programming of movements prior to their initiation.

The concept of a motor program ${ }^{2}$ derives from the observation that complex sequences of learned movements can be executed at a rate too fast to be guided by sensory feedback, or for the individual components to be each under conscious control. Studies showing that Parkinsonians can generate a voluntary motor set in response to precues, ${ }^{3}$ and can employ a predictive motor strategy in visuomotor pursuit ${ }^{4} 5$ indicate that an intact basal ganglia is not necessary Address for reprint requests: Robert D Rafal, MD, Division of
Neurology, Roger Williams General Hospital, 825 Chalkstone
Avenue, Providence, RI 02908 USA.

Received 2 September 1986 and in revised form 21 January 1987. Accepted 27 January 1987 for the voluntary assembly of motor programs. Noting that the intent or will to move seems unaffected by Parkinson's disease, Marsden ${ }^{6}$ has proposed that "the motor function of the basal ganglia is to automatically and subconsciously run the sequence of motor programs that comprise a motor plan. The programs themselves may be learned and stored elsewhere in the brain, and perhaps are assembled into a coherent plan in the premotor and frontal areas. But the initiation and automatic execution of the sequence of motor programs required to complete the motor plan of a complex motor act may depend upon the basal ganglia".

If Marsden's hypothesis is correct, the Parkinsonian's difficulty lies not in the use of information to prepare movement sequences, or to assemble the necessary motor programs, but rather at a later execution stage of motor programming which can only begin after the signal to begin the movement. This execution phase of motor programming has been studied in normal subjects in simple reaction time experiments demonstrating that the time to initiate the first component of a motor sequence (either spoken or typewritten) increases linearly as a function of the length of the entire sequence to be produced. ${ }^{7}$ One model of motor programming advanced by Sternberg et $\mathrm{al}^{7}$ to explain these results is that the abstract representation of a motor program for a learned sequence can, given a signal to "get ready", be assembled and loaded into a motor "buffer". This preparation stage, however, can be superseded by the execution phase 
only after the signal to begin the movement, at which time the motor program must be read out from its buffer to specify the spatio-temporal sequence for activation of individual muscles. As the length of the sequence increases, so does the time required to read the motor buffer, resulting in an increase in latency to initiate the first movement in the sequence (hereafter called the length effect). This length effect is the increase in RT which results from programming the sequence, and can be used to study motor programming in a way which is independent of rigidity or other peripheral factors which can also effect RT.

We have applied this paradigm to the study of sequential finger movements in Parkinsonian patients to determine whether the basal ganglia may be involved in this stage of motor programming. In separate blocks of a simple reaction time task, subjects executed a sequence of finger press responses consisting of one (index), two (index-ring) or three (indexring-middle) components. If Parkinsonians are not able to use advance information of the sequence to be executed and, instead, execute each component as a separate movement without regard to the programming requirements of the other components, then the latency to initiate the sequence with the index finger should be uninfluenced by the length of the entire sequence, and no length effect should be found. The absence of a length effect in Parkinsonians would suggest, therefore, that the basal ganglia are required for preparing this kind of motor program, or loading it into a motor buffer. According to the model of Sternberg et $a l,{ }^{7}$ the size of the length effect, that is the amount by which the latency for initiating the first component increases for each additional component in the sequence, reflects the time required to search or read the buffer. Therefore, if Parkinsonians manifest a length effect, but if the effect is relatively larger than in controls, then we could conclude that the basal ganglia dysfunction does not prevent the assembly of the motor program, but does slow the reading of the motor buffer. Finally, an analysis of inter-response latencies for non-initial movements (with the ring and middle fingers), should provide an indication whether motor programming is effective in facilitating the execution of the sequence. The function of motor programming is to permit movement sequences to be executed rapidly, as a single movement. Successful programming and execution, therefore, should permit inter-response intervals for non-initial movements to be less than RT to initiate the first component. This position effect, then, may index the effectiveness of the motor program in facilitating performance.

\section{Subjects and methods}

Eight patients with idiopathic Parkinson's disease volun- teered to participate. There were five men and three women ranging in age from 37-82 yr (mean 62). In all bradykinesia was the chief complaint, and in none was tremor severe. Two with mild bradykinesia were on no medication. Two with moderate bradykinesia were tested at the time of diagnosis before treatment was begun. Four had moderately severe bradykinesia on drug treatment at the time of testing. Controls were eight neurologically normal adults recruited from spouses of the Parkinsonian subjects and other family members of the Parkinson support group. There were two men and six women ranging in age from 45-78 yr (mean 66). After obtaining informed consent, each subject was tested in a single session consisting of six blocks: three different finger movement sequences with each the left and right hand. Subjects faced a video monitor with each of the four fingers of one hand resting on adjacent response keys on a board placed between the subject and the display. The video monitor and response keyboard were interfaced with a microcomputer which generated stimuli on the display, and recorded RT responses on line.

Each trial began, after an intertrial interval of $2000 \mathrm{~ms}$, with the appearance of a large unfilled square in the centre of the display. One second later a $50 \mathrm{~ms}$ tone sounded to further alert the subject followed, $500 \mathrm{~ms}$ later, by the appearance of a large $X$ in the centre of the box which was the "Go" signal to begin the movement sequence. In this simple RT task, the response to be made was the same for every trial in a block. Thus, subjects had information of the movement to be made which allowed them to fully prepare the sequence before the "Go" signal. In a given block the required response was one of three possible responses: (1) a one component movement consisting of a key press with the index finger [I]; (2) a two component movement (index-ring [I-R]); or a three component movement (index-ring-middle [I-R-M]). Subjects were instructed to perform as "quickly and accurately" as possible, and were encouraged to try to execute the sequences smoothly "like a single movement". Because our goal was to study the programming and execution of learned movement sequences, subjects practised each sequence thoroughly before data were collected for that movement. After a practice block of 15 trials (or more if a given subject seemed to require more practice to perform the sequence comfortably), data were collected for 35 trials in each of the six conditions. Half the subjects in each group performed the blocks in the sequence [I], then [I-R], then [I-R-M]; the other half were tested in the opposite order. Each sequence was performed with each hand before proceeding to the next sequence. Half the subjects in each group began with the right hand, and half with the left. The order of sequence length and hand was randomised across successive subjects.

Light pressure on any of the response keys activated a microswitch which recorded RT. RT for the initial movement was measured from the appearance of the "Go" signal to key press with the index finger. Inter-response latencies were also measured for the time between the index and ring finger, and between the ring and middle finger responses. Feedback was given after each trial: a high pitched tone for a correct response; a low pitched tone if the sequence were incorrect or if, for any reason, the correct response was not executed within $3000 \mathrm{~ms}$ of the "Go" signal. Only RT data from trials with correct responses were analysed. The regular 
and predictable sequence of each trial, with the visual (box) "get ready" signal followed by an auditory "get set" signal, followed by the imperative signal to "Go" occurred with the same intervals on every trial and there were no catch trials. This was done to encourage both maximum preparation and speed of performance in order to encourage programming of the sequences. To minimise the degree to which anticipatory responses could affect outcome, subjects were carefully observed for responses which occurred before the "Go" signal; and when these were observed, subjects were cautioned not to "jump the gun". Moreover, RT responses of less than $100 \mathrm{~ms}$ were excluded from analysis.

\section{Results}

The error rates in the Parkinsonians $(7 \cdot 2 \%)$ and controls $(9.9 \%)$ were not different $(\mathrm{F}[1,14]=1 \cdot 2$, $\mathrm{p}=\mathrm{NS}$ ). From observation of subjects during practice, it was evident that Parkinsonians initially found the longer sequences more difficult; they found learning the sequence more laborious, and required more practice to master each sequence. The control subjects were given just as many trials as the Parkinsonians, however, and RT data was collected only after each subject was able to execute the sequence comfortably. Thus the RT data presented below reflect performance after learning, and does not provide any indication as to whether Parkinsonians had more difficulty in learning the sequences.

After excluding RTs of less than $100 \mathrm{~ms}$, median RTs from each subject for each condition of hand (right and left), sequence length (one [I], two [I-R], and three [I-R-M] components), and component (index, ring and middle finger movement) were calculated. First, all the data were subjected to a repeated measures analysis of variance (ANOVA) with one between factor, group (Parkinson and control); and one within factor, hand (right and left). There was no difference between the two hands for the two groups. Overall, mean RT was slower for the Parkinsonians $(474 \mathrm{~ms})$ than for controls $(299 \mathrm{~ms}),(\mathrm{F}[1,14]=6 \cdot 6$, $\mathrm{p}<0.025)$.

Figure (A) shows the data for the initial movement with the index finger (solid lines) plotted as a function of the length of the sequence in which it was executed, for each of the two groups. There was, for both groups combined, a $72 \mathrm{~ms}$ increase in RT for this initial index finger movement for each subsequent movement in the required sequence. An ANOVA on these data confirmed that this length effect was highly significant $(F[2,28]=13.6, p<0.001)$; and that there was no difference in this length effect between Parkinsonians and controls $(\mathrm{F}[2,28]=0.7)$. The dashed lines of fig (A) show the increase in the interresponse latency for the second movement, that is, the time between the key press with the index finger and ring finger, as a function of the length of the entire sequence. This $39 \mathrm{~ms}$ increase in RT for the ring finger movement in the three component [I-R-M] sequence compared to the two component [I-R] sequence did not achieve statistical significance $(F[1,14]=2 \cdot 7$, $p$ $=0.12$ ); however, as shown in the fig, this effect of sequence length on the inter-response interval for the ring finger response was present in both groups, and did not differ between Parkinsonians and controls. A separate ANOVA on the index finger and ring finger RTs from the [I-R] and the [I-R-M] sequences revealed that the greater length effect in these sequences for the index finger $(83 \mathrm{~ms})$ compared with that for the ring finger $(38.5 \mathrm{~ms})$ approached statistical significance $(F[1,7]=3 \cdot 5, p=0 \cdot 10)$. The presence of a length effect for the non-initial as well as the initial response is consistent with the model of Sternberg et al ${ }^{7}$ that the motor buffer must be read to retrieve each sub-program during the execution of the program.

Figure (B) shows all the data from both groups to demonstrate that programming of the sequential movements was effective in facilitating their execution, as reflected by a systematic decrease in the inter-response latencies for the second (ring) and third (middle) components compared with the time to

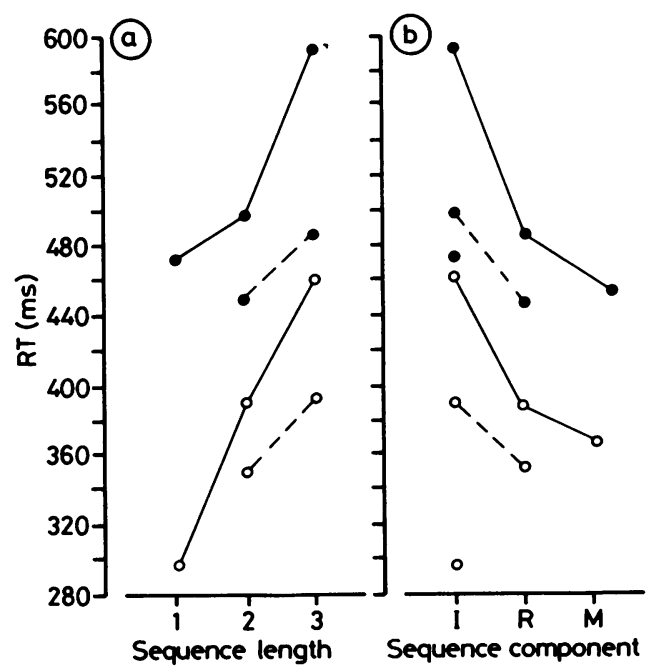

Fig (A) $R T$ in ms to initiate sequence with the index finger (solid lines), and inter-response latency between index finger and ring finger responses (dashed lines) as a function of the length of the sequence in which the responses were executed: $I-[I] ; 2-[I-R] ; 3-[I-R-M]$. Closed circles-

Parkinsonian subjects; open circles-control subjects. (B)

$R T$ in ms to execute responses with the index finger (I), ring finger $(R)$ and middle finger $(M)$ in the one component (unconnected circles), two component (dashed lines) and three component (solid lines) sequences. Closed circlesParkinsonian subjects; open circles (control subjects). 
begin the sequence with the index finger. The effect of the position of the component movement in the sequence was significant for the three component [I- $\mathrm{R}-\mathrm{M}]$ sequence shown in the solid lines $(\mathrm{F}[2,28]=$ $5.8, \mathrm{p}<0.01$ ); although it did not achieve significance for the two component [I-R] sequence (dashed lines), $(\mathrm{F}[1,14]=1.93, p=0.18)$. Again, as shown in fig (B), there was no difference in this position effect between Parkinsonians and controls. These results suggest that motor programming was effective in facilitating the efficient execution of the individual responses within integrated sequences, and that in this regard the Parkinsonians were not distinguished from the control subjects.

\section{Discussion}

The plight of the patient with Parkinson's disease calls special attention to the role of the basal ganglia in transducing the will to move into effective action. Among the cardinal symptoms of the disease, akinesia has been emphasised as being "the primary symptom of all basal ganglionic human syndromes". 8 Rigidity and tremor are important symptoms of Parkinsonism, but reflect the function of other components of the motor system released from inhibition of the basal ganglia; whereas akinesia and bradykinesia reflect the chief function of the basal ganglia, viz the facilitation of movement. ${ }^{6}$

Bradykinesia refers to slowness in execution of movements which have been initiated, and may be measured as a prolongation of movement time (MT). Electromyographic studies have indicated that it reflects a deficiency in energising muscles selected for action with sufficient force. ${ }^{9}$ However, Parkinsonians are also slow to initiate movements, resulting in an increase in reaction time (RT). This slowness in movement initiation seems closely related to akinesia, the impoverishment of spontaneous movement initiation. The clinical observation that akinesia and bradykinesia can be poorly correlated, ${ }^{10}$ and the experimental finding that RT and MT can be dissociated both within and across patients ${ }^{11}$ suggest that the pathophysiological basis for slowness in movement initiation (RT) may be distinct from that which causes slowness of movement execution (MT).

The slowness in initiating movement, which is the focus of the current investigation, is of special interest since it suggests that the basal ganglia may be involved in the programming of movements, or facilitating the implementation of these programs. Keele ${ }^{2}$ applied the term motor "program" to "a set of muscle commands that are structured before a movement sequence begins". Such programs may be assembled voluntarily, based upon an abstract representation of the desired goal of the action. The existence of these abstract representations, which have been referred to as motor "plans", 6 or "schemas" 18 has been inferred, for example, from the invariant appearance of one's signature, even when it is produced by entirely different motor programs: whether scribed by pen, with finger and hand muscles, or produced in broad strokes on a chalk board using an entirely different set of proximal muscles, the results are distinctly similar. The Parkinsonian's signature, although micrographic and effortfully produced, retains its identifiable premorbid morphology. Parkinsonians know what they want to do, and are able to voluntarily perform, albeit slowly, complex movements on command in the correct sequence and direction, and with proper relative timing. ${ }^{12}$

The difficulty of the Parkinsonian does not then derive from an inability to execute movements under conscious control; but rather from an obligation to do so. Every action requires effortfull concentration. Schwab called attention to the lack of automaticity in Parkinsonian performance which requires them to do one thing at a time. ${ }^{1314}$ If the motor plan is intact in Parkinson's disease, is it the motor program which is disrupted? The clinical phenomenon of kinesia paradoxica suggests that this is not the case: the otherwise frozen individual may, exceptionally, move briskly to catch an object unexpectedly thrown at him; or leap from his invalid bed to flee a fire. It seems, rather, that the motor program is alive and well, but somehow trapped in the Parkinsonian nervous system, inacces sible to conscious activation by the motor plan. One possibility is that the basal ganglia provide a mech anism by which the motor plan is transduced too initiate the automatic neural processes which "run" the motor program.

The experiment reported here was contrived to probe for deficits in motor programming or execution at several stages. If Parkinsonians are not able to use information of a sequence to be executed, and are therefore obliged to initiate each component of the sequence as a separate movement, then we should not have expected the length of the sequence to have influenced the time required to begin the initial component with the index finger. The index finger response would have been made without regard to the requirements of subsequent components, and its latency would not have increased as a function of the length of the entire sequence. Since a length effect was present, our Parkinsonian subjects clearly used prior information in preparing their simple RT responses. Therefore, normal basal ganglia function does not appear to be required for preparing this kind of motor program, or loading it into a motor buffer. According to the model of Sternberg $\mathrm{et} \mathrm{al}^{7}$ the magnitude of the length effect (that is, the amount by which initial RT increases for each additional component in the 
sequence) serves as an index of the time required to search or read the motor buffer. We speculated that the basal ganglia might be involved in this phase of running prepared motor programs. However, the length effect in our Parkinsonian subjects was the same size as in the control subjects, indicating that basal ganglia dysfunction does not slow the speed of searching the motor buffer. Finally, analysis of the inter-response latencies revealed that programming benefited Parkinsonians as much as controls in facilitating the execution of non-initial movements with the ring and middle finger.

In summary then, we found that Parkinsonians were slower than control subjects in initiating and executing sequential finger movements under simple RT conditions. We found no evidence, however, that this slowing was caused by an inability to use advance information in preparing motor programs, or to retrieve subprograms for execution. Whether the sequence length effect is construed as an increase in time required to search a motor buffer, ${ }^{7}$ or as the increase time required to traverse nodes between different levels of an hierarchically organised motor program, ${ }^{19}$ Parkinsonians are unimpaired in this phase of running motor programs.

According to the model of Sternberg et al, ${ }^{7}$ each unit of action within a sequence requires a search of the buffer for its subprogram. Our finding of a sequence length effect for non-initial movements is consistent with that model. The Sternberg et al model also specifies that the retrieval of each subprogram is followed by a "command" stage which is not related to the length of the sequence. Our finding that Parkinsonians were slower than controls, and slowed to the same extent for each movement in the sequence regardless of length, is consistent with the possibility that basal ganglia dysfunction slows the command stage of subprogram execution. However, this experiment was not designed to investigate this command stage per se, and does not provide any direct support for that component of the Sternberg et al model, nor for the possibility that this component is abnormal in Parkinson's disease.

Our interpretation of this experiment does assume that the sequence length effect, and the facilitation of non-initial movements compared with initial RT, do reflect motor programming. Control experiments by Sternberg et al permitted them to reject several other possible explanations for the sequence length effect, including those contingent upon short term memory capacity, readiness, or response competition. ${ }^{7}$ We should note, however, that the sequence length effect manifest in our experiment (ca 60 ms per item), is considerably larger than that found by Sternberg et al for either speech or for typewriting (ca 8-15 ms per item). We have no clear explanation for this quantitative difference in our results, and must consider whether we may not, in fact, have been measuring only motor programming effects. We have recently completed a study in which patients with cerebellar lesions were tested in the same experiment as our Parkinsonians (Inhoff, Diener and Rafal, in preparation). Cerebellar patients did manifest a deficit in the sequence length effect and in the position effect, and the severity of these deficits was related to the severity of clinical motor disability. We can, therefore, be more confident that our experiment is sensitive for measuring some aspect of motor programming and execution which requires intact cerebellar, but not basal ganglia, function.

Other studies seeking evidence for a role of the basal ganglia in motor programming have also examined whether Parkinsonians are able to use advance information to facilitate their motor performance. Initially, Parkinsonian impairment in pursuit tracking was interpreted to suggest an inability to adapt a "predictive strategy", and to implicate a deficiency in the generation of or access to an "internal representation" of the motor program. ${ }^{1516}$ Recent studies which have directly addressed this hypothesis, however, demonstrated that deficient pursuit tracking in Parkinsonians was not attributable to any failure in employing a predictive strategy. ${ }^{45}$

Another strategy for investigating whether Parkinsonians can prepare a motor program has been to provide subjects with precues instructing the preparation of a specific movement, and measuring the effects of this advance information on performance. Such studies have shown that Parkinsonians do show a facilitation of performance from such information, but several differ on whether they do so to a normal degree. Evarts et $\mathbf{l}^{11}$ measured latency for Parkinsonians to initiate an arm movement (flex or extend) after a visual signal both under simple RT conditions, in which a precue instructed which movement to prepare, and choice RT conditions in which they had no advance information. These Parkinsonian patients were quicker in the simple RT condition. They did not test any control subjects; but compared to simple vs choice RT results published in normal subjects, it appeared that their Parkinsonians perhaps benefited less in the simple RT condition. Since the motor tasks used in the two different populations were not the same, it is difficult to interpret the Evarts et al observations. Rafal et $\mathbf{l}^{3}$ tested a group of Parkinsonians on and off the effects of medication in three separate tasks, and obtained a different result. The RT difference in the drug alleviated vs unalleviated condition was greater in the two choice RT tasks than in the simple RT task. Once again, however, since the motor responses were not the same, such quantitative comparisons are hard to interpret. 
Bloxham et $\mathrm{al}^{5}$ did specifically compare simple and choice RT in the same motor task (lifting either the right or left hand in response to a word presented on a screen) in Parkinsonians and matched control subjects. The Parkinsonians were quicker in the simple than in the choice RT condition, but the difference was less than in the control subjects. Since, in the same communication, they had found no deficit in using a predictive strategy in a pursuit tracking task, but did in their RT task, Bloxham et al suggested that Parkinsonians can execute pre-programmed movements, but had difficulty in "initiating them without the help of an external trigger".

In one of the three experiments reported by Rafal et $\mathrm{al}^{3}$ (Experiment 3 ), we did specifically examine the effects of precuing on motor preparation (of a flex or extend arm movement), and found no difference in the precuing effects in Parkinsonian patients on and off medication. Because these results and those of Bloxham et $\mathrm{al}^{5}$ on the effects of precuing provide conflicting results on the effects of advance information on motor programming in Parkinson's disease, methodologic differences in the two studies should be considered:

(1) Bloxham et al compared Parkinsonians with normal control subjects; whereas Rafal et al used Parkinsonian patients as their own controls, comparing the performance of patients while symptomatic off therapy with their own performance when symptoms were ameliorated on drug therapy;

(2) Bloxham et al used an "information" (simple RT) and a "no information" (choice RT) condition. The Rafal et al task was a choice RT experiment with valid cues (that is, an "information" condition), and invalid cues (that is, a "misinformation" condition). Our experiment had no neutral cues, or "no information" condition. It is possible that our patients, off medication, did not benefit as much from the information of the precue; but, at the same time, had more cost from an invalid precue, the two effects cancelling each other out. Angel et al ${ }^{1}$ have shown that Parkinsonians are slower to reverse incorrectly prepared movement, and this may explain why we found no net precue effect differences in our Parkinsonians on and off medication;

(3) In the Rafal et al experiment, both the precue and the target were sensory signals toward which a movement had to be made. Since, in the experiment of Bloxham et al, the precues and targets were words requiring endogenous processing, the discrepancy in our findings might provide support for their hypothesis that the deficiency in Parkinsonism is related to an inability to prepare movements in the absence of external information toward which the movement must be directed. On the other hand the attempt by Bloxham et al to activate an "internal" motor set by using words as cues introduced a lexical analysis component to their task. That is, their precues not only prepared subjects to make a movement, but also to read a word. Thus, the smaller benefit of precuing in their Parkinsonian subjects may have been due to less effective lexical priming, rather than a deficiency in motor preparation. In fact, their data did suggest that their Parkinsonian subjects had made use of motor information in preparing their responses, since they showed a normal repetition effect. It should be noted that, in the experiment reported here, our Parkinsonian subjects showed clearly that their preparation of simple RT responses was influenced by an internal representation of the entire sequence to be executed; (4) While there are several possible reasons for the discrepancy between the results of Rafal et al and Bloxham et al on the effect of motor precuing, one important difference was in the motor response required in their two experiments. The precue in the experiment of Rafal et al instructed the preparation of the direction of movement of one limb (moving a lever to left or right). The precue in the Bloxham et al experiment, on the other hand, instructed the preparation of which limb was to be moved (lift the left hand or lift the right).

In a study of motor precuing in normal subjects, Rosenbaum ${ }^{17}$ showed that selection of the limb to be used, the direction of the movement, and the distance to be moved were programmed separately as distinct features of the motor program. It is certainly possible that the basal ganglia contribute to programming limb selection or of force, but not direction (studied in our previous investigation), ${ }^{3}$ or selection of finger movements in one limb (studied in the current? report). Both the precuing method, and the approach used in the current experiment, may be helpful in understanding the role of the basal ganglia in motor programming. We must now begin to isolate selected components of the motor program for independent examination.

We are grateful to Alan Wing and Ted Wright for the helpful suggestions during the conduct of this study; and to Steve Keele, David Rosenbaum and Saul Sternberg for their comments on the manuscript. Corrine Hopp assisted in preparing the manuscript. Support to E Bernstein was provided by Roger Williams General Hospital.

\section{References}

1 Angel RW, Alston W, Higgins JR. Control of movement in Parkinson's disease. Brain 1970;93:1-14.

2 Keele SW. Movement control in skilled motor performance. Psychol Bull 1968;70:387-403.

3 Rafal RD, Posner MI, Walker JA, Friedrich FJ. 
Cognition and the basal ganglia: Separating mental and motor components of performance in Parkinson's disease. Brain 1984;107:1083-94.

4 Day BL, Dick JPR, Marsden CD. Patients with Parkinson's disease can employ a predictive motor strategy. J Neurol Neurosurg Psychiatry 1984;47:1299-306.

5 Bloxham CA, Mindel TA, Frith CD. Initiation and execution of predictable and unpredictable movements in Parkinson's disease. Brain 1984;107:371-84.

6 Marsden CD. The mysterious motor function of the basal ganglia: The Robert Wartenberg Lecture. Neurology 1982;32:514-39.

7 Sternberg S, Monsell S, Knoll RL, Wright CE. The latency and duration of rapid movement sequences: Comparisons of speech and typewriting. In: Stelmach $\mathrm{GE}$, ed. Information Processing in Motor Control and Learning. New York: Academic Press, 1978.

8 Denny-Brown D, Yanagisawa $N$. The role of the basal ganglia in the initiation of movement. In: Yahr MD, ed. The Basal Ganglia. New York: Raven Press, 1976.

9 Hallett M, Koshbin S. A physiological mechanism of bradykinesia. Brain 1980;103:301-14.

10 Meyer CHA. Akinesia in Parkinsonism. Relation between spontaneous movement (other than tremor) and voluntary movements made on command. $J$ Neurol Neurosurg Psychiatry 1982;45:582-5.

11 Evarts EV, Teravainen H, Calne DB. Reaction time in Parkinson's disease. Brain 1981;104:167-86.

12 Wilson SAK. Disorders of motility and muscle tone, with special reference to the striatum. Lancet 1925;2: $1-53$.

13 Schwab RS, Chafetz ME, Walker S. Control of two simultaneous voluntary motor acts in normals and in Parkinsonism. Arch Neurol 1954;72:591-8.

14 Schwab RS, Talland GA. Performance with multiple sets in Parkinson's disease. Neurology 1964;9:65-72.

15 Cooke JD, Brown JD, Brooks VB. Increased dependence on visual information for movement control in patients with Parkinson's disease. Can J Neurol Sci 1978;5:413-5.
16 Flowers K. Lack of prediction in the motor behavior of parkinsonism. Brain 1978;101:35-52.

17 Rosenbaum DA. Human movement initiation: Specification of arm, direction and extent. J Exp Psychol Gen 1980;109:444-74.

18 Schmidt RA. A schema theory of discrete motor skill learning. Psychol Rev 1975;82:225-60.

19 Rosenbaum DA. Motor programming: A review and scheduling theory. In: Heuter $\mathbf{H}$, Kleinbeck $\mathrm{U}$, Schmidt D-H, eds. Motor Behavior: Programming, Control and Acquisition. New York: Springer-Verlag, 1985.

\section{Addendum}

Since this paper was accepted for publication, Stelmach, Worringham and Strand have published results of an experiment which systematically investigated the use of advance information in precuing of limb selection, direction and amplitude, using the Rosenbaum method (Brain 1986;109: 1179-94). They found no deficit in the utilisation of advance information in any of these tasks. They have also conducted an experiment similar to that reported here in which they measured the sequence length effect for sequences of serial taps with just the index finger. In this task they did find evidence for a programming deficit in Parkinsonians, that is, their Parkinsonian subjects did not have a sequence length effect, whereas their control subjects did (International Journal of Neuroscience, in press). Thus, although motor programming deficits do not seem to account completely for slowing of RT found in Parkinsonians, they may have deficits in programming certain types of movements, such as repetative sequences. 